\title{
Numerical Treatment of a Singularly Perturbed Boundary Value Problem Using Polynomial and Nonpolynomial Splines
}

\author{
*Aminu Audu ${ }^{1}$, A. A. Shalangwa ${ }^{1}$, David John ${ }^{1}$ \\ ${ }^{I}$ Gombe State University, Nigeria.
}

\begin{abstract}
In this study, two polynomial splines are developed and used to obtain the numerical solution of singularly perturbed two-point boundary value problems. The two polynomial splines developed are linear and non-linear polynomial splines. The applications of these splines to singularly perturbed two-point boundary value problems resulted to linear algebraic system of equations which are then solved by Gaussian elimination method to obtain the unknown constants arising from the splines used. Three singularly perturbed boundary value problems are solved.
\end{abstract}

Keywords: singularly perturbation, polynomial and non-polynomial Spline, boundary value problems and Absolute Error.

\section{Introduction}

Singularly perturbed boundary value problems are widespread in nature. Typically these problems are in various fields of applied mathematics such as fluid mechanics, elasticity, quantum mechanics, optimal control, chemical reactor theory, aerodynamics, reaction diffusion process, geophysics and many other areas. Equations of this type typically exhibit solutions with layers; that is, the domain of the differential equation contains narrow regions where the solution derivatives are extremely large. The numerical treatment of singularly perturbed differential equations gives major computational difficulties due to the presence of boundary or interior layers. Wide verities of papers and books have been published in the recent years, describing various methods for solving singularly perturbed two-point boundary value problems.

\section{Spline Methods}

The formulation of a Spline function approximation and the development of some methods for numerical solution of second-order singularly perturbed boundary value problems.

We derived the methods to solve a boundary value problem in the finite interval $[a, b]$, we partition the interval using equally spaced knots $x_{i}=a+i h, i=0,1,2, \ldots, N, x_{0}=a, x_{N}=b, h=\frac{(b-a)}{N}$, where $\mathrm{N}$ is an arbitrary positive integer. For each sub-interval $x_{i}, x_{i+1}$ for $i=0,1,2, \ldots, N-1$.

\section{Proposed Linear Polynomial Spline Function}

The proposed polynomial Spline function has the form:

$S_{i}(x)=a_{i}+b_{i}\left(x-x_{i}\right)+c_{i}\left(x-x_{i}\right)^{2}+d_{i}\left(x-x_{i}\right)^{3}, i=0,1,2, \ldots, N-1$

Where $a_{i} b_{i} c_{i}$ and $d_{i}$ are constants.

A polynomial function $S(x)$ of class $C^{2}[a, b]$ interpolates $y(x)$ at the grid point $x_{i}$ for $i=0,1,2, \ldots, N$. Let $y_{i}$ be an approximation to $y\left(x_{i}\right)$, obtained by the polynomial spline $\mathrm{S}$ passing through the points $x_{i}, y_{i}$ and $\left(x_{i+1}, y_{i+1}\right)$. The spline function (1) is not only required to satisfy the given differential equation and the associated boundary conditions at $x_{i}$ and $x_{i+1}$, but it must also satisfy the continuity of first derivatives at the common nodes $\left(x_{i}, y_{i}\right)$. We derived an expression for the coefficients of (1) in-terms of $y_{i}, y_{i+1}, M_{i}$ and $M_{i+1}$ thus,

$S_{\left(x_{i}\right)}=y_{i}, S_{\left(x_{i+1}\right)}=y_{i+1}, S^{\prime \prime}\left(x_{i}\right)=M_{i}, S^{\prime \prime}\left(x_{i+1}\right)=M_{i+1}$

From the algebraic manipulation, we obtain as from (2)

$a_{i}=y_{i}, b_{i}=\frac{\left(y_{i+1}-y_{i}\right)}{h}-\frac{h\left(M_{i+1}+2 M_{i}\right)}{6}, c_{i}=\frac{M_{i}}{2}, d_{i}=\frac{M_{i+1}-M_{i}}{6 h}$

Where $i=0,1,2, \ldots, N-1$

One sided limit of the derivative of $S(x)$ are obtain as

$S^{\prime}\left(x_{i}^{-}\right)=\frac{1}{h}\left(y_{i}-y_{i-1}\right)+\frac{h}{6}\left(M_{i}-2 M_{i-1}\right), \quad i=0,1,2, \ldots, N$

And

$S^{\prime}\left(x_{i}^{+}\right)=\frac{1}{h}\left(y_{i+1}-y_{i}\right)-\frac{h}{6}\left(M_{i+1}+2 M_{i}\right), i=0,1,2, \ldots, N-1$

Using the continuity condition of the first derivatives at $x_{i}, y_{i}$, that is

$S_{i-1}^{\prime}\left(x_{i}\right)=S_{i}^{\prime}\left(x_{i}\right)$, We obtain the following consistency relation: 
$M_{i+1}+4 M_{i}+M_{i-1}=\frac{6}{h^{2}}\left(y_{i+1}-2 y_{i}+y_{i-1}\right), i=1,2, \ldots, N-1$

\section{Singularly Perturbed Boundary Value Problems}

We consider second order singularly perturbed boundary value problem of the form:

$-\epsilon y^{\prime \prime}+q(x) y^{\prime}+p(x) y=f(x)$

$y(0)=A, y(1)=B$

Where A,B are constants and $\epsilon$ is a small positive parameter such that $0<\epsilon \leq 1$ and $q(x), p(x), f(x)$ are small bounded real functions.

\section{Linear Polynomial}

The methods developed in this section for the solution of the boundary value problem (7) are based on the Spline approximation function discussed previously. The interval $[0,1]$ is partitioned into a set of $\mathrm{N}$ equal sub-interval with length $h=\frac{1}{N}$, such that the nodal point $x_{0}=0, x_{N}=1$ and $x_{i}=i h, i=1(1) N-1$. Let $y_{i}$ been approximation to $\left(x_{i}\right)$, obtained by the segment $S(x)$ of the spline function passing through the points $\left(x_{i}, y_{i}\right)$ and $\left(x_{i+1} y_{i+1}\right)$, where $S(x)$ is as defined by (1).

We considered two cases of (7)

Case (a): when $q(x)=0$

Now, setting $=x_{i}$, in (7) and making use of (2), we have

$M_{i}=\frac{p\left(x_{i}\right)}{\epsilon} y_{i}-\frac{1}{\epsilon} f_{i}$

It follows also that

$M_{i-1}=\frac{p\left(x_{i-1}\right)}{\epsilon} y_{i-1}-\frac{1}{\epsilon} f_{i-1}$

And

$M_{i+1}=\frac{p\left(x_{i+1}\right)}{\epsilon} y_{i+1}-\frac{1}{\epsilon} f_{i+1}$

On using (9)-(11) in the consistency relation (6), we obtain

$\left(h^{2} p_{i-1}-6 \epsilon\right) y_{i-1}+4\left(h^{2} p_{i}+3 \epsilon\right) y_{i}+\left(h^{2} p_{i+1}-6 \epsilon\right) y_{i+1}=h^{2}\left(f_{i-1}+4 f_{i}+f_{i+1}\right)$

(12)

$$
i=1,2, \ldots, N-1
$$

Thus, (12) together with the two boundary conditions (8) gives rise to a tridiagonal set of $(n+1)$ algebraic equations which are solved by Gaussian elimination method for the $(n+1)$ unknown $y_{i}, i=0,1,2, \ldots, N$.

The local truncation error of (12) is given by

$T_{i}=\left[h^{2} p\left(x_{i-1}\right)-6 \epsilon\right] y\left(x_{i-1}\right)+4\left[h^{2} p\left(x_{i}\right)+3 \epsilon\right] y\left(x_{i}\right)+\left[h^{2} p\left(x_{i+1}\right)-6 \epsilon\right] y\left(x_{i+1}\right)-h^{2} f\left(x_{i-1}\right)-$

$4 h^{2} f\left(x_{i}\right)-h^{2} f\left(x_{i+1}\right)$

Case (b): when $q(x) \neq 0$

Now, setting $x=x_{i}$ in (7) and making use of (2)

$M_{i}=\frac{q\left(x_{i}\right)}{\epsilon} y_{i}^{\prime}+\frac{p\left(x_{i}\right)}{\epsilon} y_{i}-\frac{1}{\epsilon} f_{i}$

It follows also that

$M_{i-1}=\frac{q\left(x_{i-1}\right)}{\epsilon} y_{i-1}^{\prime}+\frac{p\left(x_{i-1}\right)}{\epsilon} y_{i-1}-\frac{1}{\epsilon} f_{i-1}$

$M_{i+1}=\frac{q\left(x_{i+1}\right)}{\epsilon} y_{i+1}^{\prime}+\frac{p\left(x_{i+1}\right)}{\epsilon} y_{i+1}-\frac{1}{\epsilon} f_{i+1}$

Substituting (14), (15) and (16) in the consistency relation (6) and using the following approximations for finite difference of first order derivatives of $\mathrm{y}$,

$y_{i}^{\prime}=\frac{y_{i+1}-y_{i-1}}{2 h}+0\left(h^{2}\right)$

$y_{i+1}^{\prime}=\frac{3 y_{i+1}-4 y_{i}+y_{i-1}}{2 h}+0\left(h^{2}\right)$

$y_{i-1}^{\prime}=\frac{-y_{i+1}+4 y_{i}-3 y_{i-1}}{2 h}+0\left(h^{2}\right)$

We obtain.

$$
\begin{aligned}
\left(6 \epsilon+\frac{p_{i+1}}{2 h}-\frac{2 p_{i}}{h}\right. & \left.+q_{i-1}-\frac{3 p_{i-1}}{2 h}\right) y_{i-1}+\left(-12 \epsilon-\frac{2 p_{i-1}}{h}+4 q_{i-1}+\frac{2 p_{i-1}}{h}\right) y_{i} \\
& +\left(6 \epsilon+\frac{3 p_{i+1}}{2 h}+\frac{2 p_{i}}{h}+q_{i+1}-\frac{p_{i-1}}{2 h}\right) y_{i+1}=h^{2}\left(f_{i-1}+4 f_{i}+f_{i+1}\right)
\end{aligned}
$$

$i=1,2, \ldots, N-1$

Thus, (18) together with the two boundary conditions (8) gives rise to a tridiagonal set of $(n+1)$ algebraic equations which are solved by Gaussian elimination method for the $(n+1)$ unknowns $y_{i}, i=0,1,2, \ldots, N$ 
Numerical Treatment of a Singularly Perturbed Boundary Value Problem Using Polynomial and ..

Proposed Nonlinear Polynomial Spline Function

The nonlinear polynomial Spline function $S(x, \tau)=S(x)$ satisfying in $\left[x_{i}, x_{i+1}\right]$ the differential equation $S^{\prime \prime}(x)-\tau S(x)=S^{\prime \prime}\left(x_{i}\right)-\tau S\left(x_{i}\right) \frac{\left(x_{i+1}-x\right)}{h}+S^{\prime \prime}\left(x_{i+1}\right)-\tau S\left(x_{i+1}\right) \frac{\left(x-x_{i}\right)}{h} ;(19)$

Where $S\left(x_{i}\right)=y_{i}$ and $\tau>0$ is termed as cubic spline in tension. Solving the linear second-order differential (19) and determining the arbitrary constant from the interpolatory condition (2), we get after writing $\lambda=h \tau^{\frac{1}{2}}$.

$S(x)=\frac{h^{2}}{\lambda^{2} \sin h \lambda}\left[M_{i+1} \sin h \frac{\lambda\left(x-x_{i}\right)}{h}+M_{i} \sin h \frac{\lambda\left(x_{i+1}-x\right)}{h}\right]-\frac{h^{2}}{\lambda^{2}}\left[\frac{\left(x-x_{i}\right)}{h}\left(M_{i+1}-\frac{\lambda^{2}}{h^{2}} y_{i+1}\right)-\frac{\left(x_{i+1}-x\right)}{h}\left(M_{i}-\frac{\lambda^{2}}{h^{2}} y_{i}\right)\right]$

(20)

Differentiating (20) and letting $x \rightarrow x_{i}$ i.e.one sided limit of derivatives of $S(x)$, we obtain

$S^{\prime}\left(x_{i}^{+}\right)=\frac{y_{i+1}-y_{i}}{h}-\frac{h}{\lambda^{2}}\left[\left(1-\frac{\lambda}{\sin h \lambda}\right) M_{i+1}+(\lambda \cot h \lambda-1) M_{i}\right]$

Considering the interval $\left[x_{i+1}, x_{i}\right]$ and processing similarly, we obtain

$S^{\prime}\left(x_{i}^{-}\right)=\frac{y_{i}-y_{i-1}}{h}+\frac{h}{\lambda^{2}}\left[(\lambda \cot h \lambda-1) M_{i}+\left(1-\frac{\lambda}{\sin h \lambda}\right) M_{i-1}\right]$

Equating the left and right hand derivatives at $x_{i}, S_{i-1}^{\prime}\left(x_{i}\right)=S_{i}^{\prime}\left(x_{i}\right)$, i.e continuity condition, we have $\frac{y_{i}-y_{i-1}}{h}+\frac{h}{\lambda}\left[(\lambda \cot h \lambda-1) M_{i}+\left(1-\frac{\lambda}{\sin h \lambda}\right) M_{i+1}\right]=\frac{y_{i+1}-y_{i}}{h}-\frac{h}{\lambda^{2}}\left[\left(1-\frac{\lambda}{\sin h \lambda}\right) M_{i+1}+(\lambda \cot h \lambda-1) M_{i}\right]$ (23)

This leads to the consistency relation

$h^{2}\left(\lambda_{1} M_{i-1}+2 \lambda_{2} M_{i}+\lambda_{1} M_{i+1}\right)=y_{i-1}-2 y_{i}+y_{i+1}$

Where $\lambda_{1}=\frac{1}{\lambda^{2}}\left(1-\frac{\lambda}{\sin h \lambda}\right), \lambda_{2}=\frac{1}{\lambda^{2}}(\lambda \cot h \lambda-1), i=1(1) 1 N-1$.

\section{Nonlinear Polynomial}

For a numerical solution of the boundary value problem (7) the interval [0,1] is divided into a set of grid points with step length $h=\frac{(b-a)}{N}, \mathrm{~N}$ being a positive integer. The spline approximation on $[0,1]$ that consist of the nodal point $x_{0}=a, x_{N}=b, x_{i}=a+i h, i=1(1) 1 N-1$.

We consider two cases of (7)

Case (a): when $q(x)=0$

Now, setting $=x_{i}$, in (7)

$-\epsilon y^{\prime \prime}\left(x_{i}\right)+p\left(x_{i}\right) y\left(x_{i}\right)=f\left(x_{i}\right)$

Now, making use of (2) and substituting (9), (10) and (11) into consistency relation (24), we obtain $\left(\lambda_{1} h^{2} p_{i-1}-\epsilon\right) y_{i-1}+2\left(\lambda_{2} h^{2} p_{i}+\epsilon\right) y_{i}+\left(\lambda_{1} h^{2} p_{i+1}-\epsilon\right) y_{i+1}=h^{2}\left(\lambda_{1} f_{i-1}+2 \lambda_{2} f_{i}+\lambda_{1} f_{i+1}\right), \quad i=$ 1(1) $1 N-1$

Thus, (26) together with the two boundary conditions (8) gives a tridiagonal system of $(n+1)$ algebraic equations which are solved by Gaussian elimination method for $(n+1)$ unknown $y_{i}, i=0,1,2, \ldots, N$.

The local truncation error of (26) is given by

$T_{i}=\left[\lambda_{1} h^{2} p\left(x_{i-1}\right)-\epsilon\right] y\left(x_{i-1}\right)+2\left[\lambda_{2} h^{2} p\left(x_{i}\right)+\epsilon\right] y\left(x_{i}\right)+\left[\lambda_{1} h^{2} p\left(x_{i+1}\right)-\epsilon\right] y\left(x_{i+1}\right)-\lambda_{1} h^{2} f\left(x_{i-1}\right)-$ $2 \lambda_{2} h^{2} f\left(x_{i}\right)-\lambda_{1} h^{2} f\left(x_{i+1}\right)$

Expanding in Taylor's series about $x_{i}$, we obtain

$T_{i}=h^{2}\left[2\left(\lambda_{1}+\lambda_{2}\right)-1\right] \epsilon y^{(2)}\left(x_{i}\right)+\left(\lambda_{1}-\frac{1}{12}\right) \epsilon h^{4} y^{(4)}\left(x_{i}\right)+\left[\frac{\lambda_{1}}{12}-\frac{1}{360}\right] \epsilon h^{6} y^{(6)}\left(x_{i}\right)+0\left(h^{8}\right) \quad i=1,2, \ldots, N$

Thus, the analysis of the methods shows that it has second-order convergence for arbitrary choices of $\lambda_{1}$ and $\lambda_{2}$ provided that $\lambda_{1}+\lambda_{2}=\frac{1}{2}$ and fourth-order convergence for $\lambda_{1}=\frac{1}{12}$ and $\lambda_{2}=\frac{5}{12}$.

Case (b): when $q(x) \neq 0$

Now, setting $x=x_{i}$ in (7) and making use of (2), (14), (15) and (16) into the consistency relation (24) and the finite difference approximation of first order derivative of $y$ i.e (17), we have

$\left(-\epsilon+\lambda_{1} h^{2} p_{i-1}-\frac{3}{2} \lambda_{1} h q_{i-1}-\lambda_{2} h q_{i}+\frac{1}{2} \lambda_{1} h q_{i+1}\right) y_{i-1}+\left(2 \epsilon+2 \lambda_{2} h^{2} p_{i}+2 \lambda_{1} h q_{i-1}-2 \lambda_{1} h p_{i+1}\right) y_{i}+$
$\left(-\epsilon+\lambda_{1} h^{2} p_{i+1}-\frac{1}{2} \lambda_{1} h q_{i-1}+\lambda_{2} h q_{i}+\frac{3}{2} \lambda_{1} h q_{i+1}\right) y_{i+1}=h^{2}\left(\lambda_{1} f_{i-1}+2 \lambda_{2} f_{i}+\lambda_{1} f_{i+1}\right)$

$i=1(1) N-1$.

Thus, (29) together with the boundary condition (8) gives rise to a tridiagonal system of $(n+1)$ algebraic algebraic equations which can be solve by Gaussian elimination method for $(n+1)$ unknown $y_{i}, i=$ $0,1,2, \ldots, N$. 
Numerical Treatment of a Singularly Perturbed Boundary Value Problem Using Polynomial and ..

\section{Example (1)}

Consider the following problem

$-\epsilon y^{\prime \prime}+y=-\cos ^{2}(\pi x)-2 \epsilon \pi^{2} \cos (2 \pi x) ; \quad y(0)=y(1)=0$

The exact solution:

$$
y(x)=\frac{\exp ^{\left(\frac{x-1}{\sqrt{\epsilon}}\right)}+\exp ^{\frac{-x}{\sqrt{\epsilon}}}}{1+\exp ^{\frac{-1}{\sqrt{\epsilon}}}}-\cos ^{2}(\pi x)
$$

Example (2)

$-\epsilon y^{\prime \prime}+1+x(1-x) y=1+x(1-x)+2 \sqrt{\epsilon}-x^{2}(1-x) \exp ^{\frac{-(1-x)}{\sqrt{\epsilon}}}+2 \sqrt{\epsilon}-x\left(1-x^{2}\right) \exp ^{\frac{-x}{\sqrt{\epsilon}}}$

$y(0)=y(1)=0$

The exact solution:

$y(0)=1+(x-1) \exp ^{\left(\frac{-x}{\sqrt{\epsilon}}\right)}-x \exp ^{\frac{-(1-x)}{\sqrt{\epsilon}}} ;$

Example (3)

$-\epsilon y^{\prime \prime}+y=-x$,

$$
y(0)=y(1)=0
$$

The exact solution:

Note:

$$
y(x)=\frac{x \exp ^{\frac{1}{\sqrt{\epsilon}}}-x \exp ^{\frac{-1}{\sqrt{\epsilon}}}+\exp ^{\frac{-x}{\sqrt{\epsilon}}}-\exp ^{\frac{x}{\sqrt{\epsilon}}}}{\exp ^{\frac{1}{\sqrt{\epsilon}}}-\exp ^{\frac{-1}{\sqrt{\epsilon}}}}
$$

CLP $=$ Computed Linear Polynomial

$\mathrm{CNLP}=$ Computed Nonlinear Polynomial

Table 1: Example 1 Solution $N=16$ with $\epsilon=\frac{1}{16}$

\begin{tabular}{|l|l|l|l|l|l|}
\hline $\mathrm{x}$ & Exact & Approximate CLP & Error CLP & Approximate CNLP & Error CNLP \\
\hline 0.00 & 0.0000000000 & 0.0000000000 & $0.0000 e^{+00}$ & 0.0000000000 & $0.0000 e^{+00}$ \\
\hline 0.06 & -0.1740519068 & -0.1740095234 & $4.2383 e^{-05}$ & -0.1740585259 & $6.6191 e^{-06}$ \\
\hline 0.12 & -0.2282776718 & -0.2288350821 & $5.5741 e^{-04}$ & -0.2282834696 & $5.7978 e^{-06}$ \\
\hline 0.19 & -0.1893944408 & -0.1910730187 & $1.6786 e^{-03}$ & -0.1893937460 & $6.9480 e^{-07}$ \\
\hline 0.25 & -0.0898457280 & -0.0929603531 & $3.1146 e^{-03}$ & -0.0898350631 & $1.0665 e^{-05}$ \\
\hline 0.31 & 0.0354714189 & 0.0308600796 & $4.6113 e^{-03}$ & 0.0354931447 & $2.1726 e^{-05}$ \\
\hline 0.38 & 0.1532788853 & 0.1473690930 & $5.9098 e^{-03}$ & 0.1533104705 & $3.1585 e^{-05}$ \\
\hline 0.44 & 0.2360916672 & 0.2293031586 & $6.7885 e^{-03}$ & 0.2361300087 & $3.8342 e^{-05}$ \\
\hline 0.50 & 0.2658022288 & 0.2587033839 & $7.0988 e^{-03}$ & 0.2658429688 & $4.0740 e^{-05}$ \\
\hline 0.56 & 0.2360916672 & 0.2293031586 & $6.7885 e^{-03}$ & 0.2361300087 & $3.8342 e^{-05}$ \\
\hline 0.62 & 0.1532788853 & 0.1473690930 & $5.9098 e^{-03}$ & 0.1533104705 & $3.1585 e^{-05}$ \\
\hline 0.69 & 0.0354714189 & 0.0308600796 & $4.6113 e^{-03}$ & 0.0354931447 & $2.1726 e^{-05}$ \\
\hline 0.75 & -0.0898457280 & -0.0929603531 & $3.1146 e^{-03}$ & -0.0898350631 & $1.0665 e^{-05}$ \\
\hline 0.81 & -0.1893944408 & -0.1910730187 & $1.6786 e^{-03}$ & -0.1893937460 & $6.9480 e^{-07}$ \\
\hline 0.88 & -0.2282776718 & -0.2288350821 & $5.5741 e^{-04}$ & -0.2282834696 & $5.7978 e^{-06}$ \\
\hline 0.94 & -0.1740519068 & -0.1740095234 & $4.2383 e^{-05}$ & -0.1740585259 & $6.6191 e^{-06}$ \\
\hline 1.00 & 0.0000000000 & 0.0000000000 & $0.0000 e^{+00}$ & 0.0000000000 & $0.0000 e^{+00}$ \\
\hline
\end{tabular}

Table 2: Example 2 Solution $N=16$ with $\epsilon=\frac{1}{16}$

\begin{tabular}{|l|l|l|l|l|l|}
\hline $\mathrm{X}$ & Exact & Approximate CLP & Error CLP & Approximate CNLP & Error CNLP \\
\hline 0.00 & 0.0000000000 & 0.0000000000 & $0.0000 e^{+00}$ & 0.0000000000 & $0.0000 e^{+00}$ \\
\hline 0.06 & 0.2684044068 & 0.2694052773 & $1.0009 e^{-03}$ & 0.2684083629 & $3.9562 e^{-06}$ \\
\hline 0.12 & 0.4655109998 & 0.4670703220 & $1.5593 e^{-03}$ & 0.4655171911 & $6.1912 e^{-06}$ \\
\hline 0.19 & 0.6089320119 & 0.6107696509 & $1.8376 e^{-03}$ & 0.6089393415 & $7.3296 e^{-06}$ \\
\hline 0.25 & 0.7116436520 & 0.7135931376 & $1.9495 e^{-03}$ & 0.7116514622 & $7.8102 e^{-06}$ \\
\hline 0.31 & 0.7830504955 & 0.7850229922 & $1.9495 e^{-03}$ & 0.7830584298 & $7.9343 e^{-06}$ \\
\hline 0.38 & 0.8297617754 & 0.8317197029 & $1.9579 e^{-03}$ & 0.8297696770 & $7.9016 e^{-06}$ \\
\hline 0.44 & 0.8561399961 & 0.8580776892 & $1.9377 e^{-03}$ & 0.8561478329 & $7.8369 e^{-06}$ \\
\hline 0.50 & 0.8646647168 & 0.8665938389 & $1.9291 e^{-03}$ & 0.8646725249 & $7.8081 e^{-06}$ \\
\hline 0.56 & 0.8561399961 & 0.8580776892 & $1.9377 e^{-03}$ & 0.8561478329 & $7.8369 e^{-06}$ \\
\hline 0.62 & 0.8297617754 & 0.8317197029 & $1.9579 e^{-03}$ & 0.8297696770 & $7.9016 e^{-06}$ \\
\hline 0.69 & 0.7830504955 & 0.7850229922 & $1.9725 e^{-03}$ & 0.7830584298 & $7.9343 e^{-06}$ \\
\hline 0.75 & 0.7116436520 & 0.7135931376 & $1.9495 e^{-03}$ & 0.7116514622 & $7.8102 e^{-06}$ \\
\hline 0.81 & 0.6089320119 & 0.6107696509 & $1.8367 e^{-03}$ & 0.6089393415 & $7.3296 e^{-06}$ \\
\hline 0.88 & 0.6089320119 & 0.2694052773 & $1.5593 e^{-03}$ & 0.4655171911 & $6.1912 e^{-06}$ \\
\hline 0.94 & 0.2684044068 & 0.2694052773 & $1.0000 e^{-03}$ & 0.2684083629 & $3.9562 e^{-06}$ \\
\hline 1.00 & 0.0000000000 & 0.0000000000 & $0.0000 e^{+00}$ & 0.0000000000 & $0.0000 e^{+00}$ \\
\hline
\end{tabular}


Table 3: Example 3 Solution $N=16$ with $\epsilon=\frac{1}{16}$

\begin{tabular}{|l|l|l|l|l|l|}
\hline $\mathrm{x}$ & Exact & Approximate CLP & Error CLP & Approximate CNLP & Error CNLP \\
\hline 0.00 & 0.0000000000 & 0.0000000000 & $0.0000 e^{+00}$ & 0.0000000000 & $0.0000 e^{+00}$ \\
\hline 0.06 & -0.0532433828 & -0.0533155216 & $7.2139 e^{-05}$ & -0.0532436069 & $2.2404 e^{-07}$ \\
\hline 0.12 & -0.1059052075 & -0.1060509709 & $1.4576 e^{-04}$ & -0.1059056603 & $4.5274 e^{-07}$ \\
\hline 0.19 & -0.1573673790 & -0.1575896395 & $2.2226 e^{-04}$ & -0.1573680693 & $6.9028 e^{-07}$ \\
\hline 0.25 & -0.2069364324 & -0.2072392326 & $3.0280 e^{-04}$ & -0.2069373727 & $9.4028 e^{-07}$ \\
\hline 0.31 & -0.2537999655 & -0.2541881457 & $3.8818 e^{-04}$ & -0.2538011707 & $1.2052 e^{-06}$ \\
\hline 0.38 & -0.2969755985 & -0.2974542048 & $4.7861 e^{-04}$ & -0.2969770842 & $1.4857 e^{-06}$ \\
\hline 0.44 & -0.3352492549 & -0.3358226348 & $5.7338 e^{-04}$ & -0.3352510344 & $1.7794 e^{-06}$ \\
\hline 0.50 & -0.3670988856 & -0.3677693364 & $6.7045 e^{-04}$ & -0.3671009658 & $2.0802 e^{-06}$ \\
\hline 0.56 & -0.3905988443 & -0.3913646277 & $7.6578 e^{-04}$ & -0.3906012196 & $2.3753 e^{-06}$ \\
\hline 0.62 & -0.4032989066 & -0.4041513692 & $8.5246 e^{-04}$ & -0.4033015501 & $2.6435 e^{-06}$ \\
\hline 0.69 & -0.4020703318 & -0.4029897760 & $9.1944 e^{-04}$ & -0.4020731821 & $2.8503 e^{-06}$ \\
\hline 0.75 & -0.3829092956 & -0.3838591161 & $9.4982 e^{-04}$ & -0.3829122392 & $2.9436 e^{-06}$ \\
\hline 0.81 & -0.3406853455 & -0.3416037688 & $9.1842 e^{-04}$ & -0.3406881909 & $2.8455 e^{-06}$ \\
\hline 0.88 & -0.2688190736 & -0.2696076070 & $7.8853 e^{-04}$ & -0.2688215159 & $2.4423 e^{-06}$ \\
\hline 0.94 & -0.1588687578 & -0.1593761360 & $5.0738 e^{-04}$ & -0.1588703288 & $1.5710 e^{-06}$ \\
\hline 1.00 & 0.0000000000 & 0.0000000000 & $0.0000 e^{+00}$ & 0.0000000000 & $0.0000 e^{+00}$ \\
\hline
\end{tabular}

The above examples can be solve using different values of $\mathrm{N}=16,32,64,128,256$ and $\epsilon=\frac{1}{16}, \frac{1}{32}, \frac{1}{64}, \frac{1}{128}, \frac{1}{256}$. The maximum absolute errors defined by Absolute Error $=\underbrace{\max }_{a \leq x \leq b}\left|\overline{y_{i}}-y_{i}\right|$ where $\overline{y_{i}}$, is the exact solution is and $y_{i}$ is the approximate solution.

The results presented above are linear polynomial and nonlinear polynomial splines together with their absolute errors, which show that the nonlinear polynomial Spline is better than the linear polynomial Spline in-terms of accuracy as compared with the exact solution.

\section{References}

[1]. Agarwal R., O’Regan D. (2009): Ordinary and partial differential equations with special functions, Fourier series and boundary value problems. Springer, New York.

[2]. Fazal-i-Haq (2009): Numerical solution of boundary value and initial boundary value problems using spline functions, PH.D thesis, Ghulam Ishaq Khan Institute of Engineering Sciences and Technology.

[3]. Islam,S. Khan, M.A., Tirmizi, S.I.A. and Twizell, E.H. (2005): A non-polynomial spline approach to the solution of a system of third-order boundary value problems using non-polynomial spline, Applied Mathematics and Computation, 168(1), 152-163.

[4]. Taiwo, O.A and Ogunlaran, O.M. (2011): A non-polynomial spline method for solving linear fourth order boundary value problems, International Journal of Physical Sciences, 6(13), 3246-3254.

[5]. Tariq, A. Arshad, Khan (2002): A spline method for second-order singularly perturbed boundary value problems, Journal of Computational and Applied Mathematics, 147, 445-452.

[6]. Kadalbajoo, M.K, Bawa, R.K. (1996): Variable mesh difference scheme for singularly perturbed boundary value problems using splines, Journal of Optimization Theory and Applied, 90(2) 405-416. 